\title{
AN ADAPTIVE THRESHOLD SEGMENTATION FOR DETECTION OF NUCLEI IN CERVICAL CELLS USING WAVELET SHRINKAGE ALGORITHMS
}

\author{
B.Savitha, Dr. P. Subashini \\ Ph.D Scholar, Associate Professor \\ Department of Computer Science \\ Avinashilingam Institute for Home Science and Higher Education for Women \\ University, \\ Coimbatore, India. \\ *savibalu36@gmail.com
}

\begin{abstract}
PAP smear test is the most efficient and easy procedure to detect any abnormality in cervical cells. It becomes difficult for the cytologist to analyse a large set of PAP smear test images when there is a rapid increase in the incidence of cervical cancer. On the replacement, image analysis could swap manual interpretation. This paper proposes a method for the detection of cervical cells in pap smear images using wavelet based thresholding. First, Wiener filter is used for smoothing to suppress the noise and to improve the contrast of the image. Second, optimal threshold is been obtained for segmenting the cell by various Wavelet shrinkage techniques like VisuShrink, BayesShrink and SureShrink thresholding which segment the foreground from the background and detect cell component like nucleus from the clustered cell images. From the results, it is proved that the performance of the adaptive Wiener filter with combination of SureShrink thresholding performs better in terms of threshold values and Mean Squared Error than the other comparative methods. The succeeding research work can be carried out based on the size of the segmented nucleus which therefore helps in differentiating abnormality among the cells.
\end{abstract}

\section{KEYWORDS}

Pap smear test, Wiener filter, VisuShrink, BayesShrink and SureShrink thresholding.

\section{INTRODUCTION}

To date, one of the most common cancers among women worldwide is Cervical Cancer. Cancer of the cervix is primarily caused by human papillomavirus (HPV) infection. Cervical cancer has no apparent symptoms like lumps, pain in the early stage. At later stage only, it causes discomfort in the lower abdominal. Though, cervical cancer is dangerous it can be prevented if it is detected and treated early with the help of PAP smear test. Pathologists carry out this analysis by visual examination of the images. However, the task is time consuming, expensive, and tedious. In order to overcome these problems, medical images made the doctors to analyse the internal portions of the body for painless diagnosis. A common shortcoming in the imaging system is unwanted non linearity in the sensor and the display system that produces noises in the images. Image denoising has become a very vital exercise for the complete system until the end of diagnoses. In this

David C. Wyld (Eds) : ICCSEA, SPPR, CSIA, WimoA - 2013

pp. 321-327, 2013. (C) CS \& IT-CSCP 2013

DOI : $10.5121 /$ csit.2013.3533 
research work, noise identified in the microscopic images is poisson noise. With this, it is proposed to combine pre-processing with segmentation that contributes segmented nucleus from the denoised image.

The paper is structured as follows, the pre-processing procedure adopted in the work is detailed in the section 2, section 3 discusses the segmentation procedure, section 4 discusses the experimental results for the taken image dataset and section 5 gives the conclusion and followed by references used.

\section{PRe PRoCessing}

The pre-processing stage is mandatory for noise removal, extracting background and for sharpening the region of interest. The pap smear images are coloured optical images which are of reduced quality due to stains used to colour the cells and uneven lighting across the field of view. In order to remove the noise, denoising is carried out for the original images using various filtering methods like Wiener filter, Lucy-Richardson filter and Regularized filter. For the objective evaluation, PSNR and MSE values for each denoised image has been calculated.

\subsection{Poisson Noise}

Quality improvement of the corrupted medical image is essential. The medical data represent by photon images has a tendency to be degraded by Poisson noise [8]. Poisson noise is signal dependent, which is frequently seen in photon images. The value of the noise is proportional to the original image values. The noise models are described as

$$
d(m, n) \sim \frac{1}{\wedge} \text { poisson }\left\{\wedge_{\circ}(m, n)\right\}
$$

Let $\curlywedge=0.5$, where $\curlywedge_{\circ}(m, n)$ and $d(m, n)$ represents the pixel values of the original and resultant images, respectively. The resultant image is generated by multiplying the pixel values of original image by $<$ and by using these as the input to a random number generator which returns Poisson distributed values. The total amount of noise depends on $ᄉ$.

\subsection{Lucy-Richardson deconvolution filter}

"Lucy-Richardson deconvolution [8], is an iterative process for improving an underlying image that have been blurred by a known point spread function. Pixel in the observed image can be represented in terms of the point spread function and the latent image as

$$
d_{i}=\sum_{j} p_{i j} u_{j} \quad \quad \cdots \cdots \cdots
$$

where, $p_{i j}$ is the point spread function (the fraction of light coming from true location, $\mathrm{j}$ that $\mathrm{s}$

observed at position), $u_{j}$ is the pixel value at location $j$ in the latent image, and $d_{i}$ is the observed value at pixel location $i$ ".

\subsection{Regularized filter}

Regularized deconvolution [8] can be used efficiently when constraints are applied on the recovered image (e.g., smoothness) and when the limited information is known about the additive 
noise. The blurred and noisy image is restoring by a constrained least square restoration algorithm that uses a regularized filter.

\subsection{Wiener Filter}

There are two dominant techniques to reduce the noise level in the image; Wiener filtering techniques and Wavelet thresholding techniques. Wiener filter [1] provides the best restored image with respect to the square error averaged over the original image and the noise.

The above assessment techniques are implemented on Pap smear images. Fig.1 and Fig.2 contains the subjective comparison between various filtering methods, and comparison between various shrinkage based threshold methods using adaptive wiener filter.

\begin{tabular}{|c|c|c|c|}
\hline $\begin{array}{l}\text { Original } \\
\text { Image }\end{array}$ & $\begin{array}{l}\text { Wiener } \\
\text { Filter }\end{array}$ & $\begin{array}{l}\text { Lucy- } \\
\text { Richardson } \\
\text { Filter }\end{array}$ & $\begin{array}{l}\text { Regularized } \\
\text { Filter }\end{array}$ \\
\hline & & & 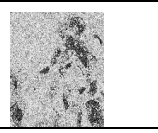 \\
\hline & & & \\
\hline & & & \\
\hline Bons & & 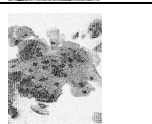 & \\
\hline 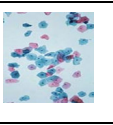 & tis & 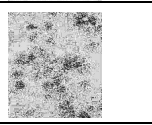 & $\begin{array}{l}2=2 \\
s^{2}-x^{2}\end{array}$ \\
\hline
\end{tabular}

Fig.1: Shows the subjective evaluation of Wiener filter, Lucy-Richardson filter and Regularized filter

\section{SEGMENTATION}

In cytological images, segmentation of cells is a fundamental subject of quantitative analysis. Because, the abnormality characteristics of cancer cells are contained in cell nucleus only, so the accurate determination of cell nuclei area is important for the correct diagnosis decision.

\subsection{Thresholding}

Thresholding is one of the widely used methods for image segmentation. In this paper, optimal threshold value is obtained from the wavelet shrinkage or threshold algorithms like visuShrink, BayesShrink and SureShrink thresholding algorithm and this iterative thresholding method is used to segment the nucleus from the Papsmear image. The key parameter in the thresholding process is the selection of the threshold value. In this method, the threshold value $\mathrm{T}$ is iteratively calculated and the pixels with gray values less than $\mathrm{T}$ are classified as object pixels, and pixels with gray values greater than $\mathrm{T}$ are classified as background pixels. 


\subsubsection{VisuShrink Thresholding}

Donoho \& Johnston [5] derived Universal threshold and uses a threshold value ' $t$ ' that is proportional to the standard deviation of the noise. Following is the algorithm of VisuShrink Thresholding,

Step 1: Let, the noisy vector $\mathbf{y}=\mathbf{v}+\mathrm{e}$, where $\mathbf{e}$ is Gaussian white noise

Step 2: Minimize the mean squared error.

Step 3: calculate t,

$$
t=\sigma \sqrt{2 \log n} \quad \ldots \ldots \ldots
$$

Where, $\sigma$ is the noise variance present in the image, $\mathrm{n}$ is the size or number of values in $\mathrm{y}$.

Step 4: As $\mathrm{N}->\infty$ the Median Absolute Deviation of the high pass values converges to $.6745 \sigma$, so the estimate for $\sigma$ is $\operatorname{MAD}(d) / .6745$.

\subsubsection{SureShrink Thresholding}

"A threshold chooser based on Stein's Unbiased Risk Estimator (SURE) that removes the error obtained $\boldsymbol{K}^{\text {univ }}$ which depends on the size of the data set. Following is the algorithm of SureShrink Thresholding,

Step 1: Minimize the mean squared error by minimizing the function

$$
f(\lambda)=N+\|g(x)\| 2+2 \Sigma d / d x k(g k(x))
$$

Where $g_{k}(\mathrm{x})$ is the threshold function minus the value for each value of $x_{k}, \quad \mathrm{k}=1,2 \ldots \mathrm{N}$

Step 2: Once this value for $\kappa^{\text {sure }}$ has been chosen, use it in the original threshold function to shrink the high pass portion and continue the process of denoising.

Step 3: With the linear soft threshold function, the function simplifies nicely as $f(\lambda)=N-2 \cdot \#$ $\left\{\mathrm{k}:\left|x_{k}\right| \leq \lambda\right\}+\Sigma \min \left(x_{k}^{z}, \boldsymbol{\alpha}^{2}\right)$. This gives different functions of $\lambda$ between $\mathrm{xk}$ and $\mathrm{xk}+1$ with a minimum at xk each time".

\subsubsection{BayesShrink Thresholding}

The goal of the Bayes shrink [6] thresholding method is to minimize the Bayesian risk. Like the SureShrink thresholding procedure, it adapts smoothness. Following is the algorithm of BayesShrink Thresholding,

Step 1: Express the observation model as $y=x+v$

Where, $\mathrm{y}$ is the wavelets transform of the degraded image, $\mathrm{x}$ is the wavelet transform of the original image, and $\mathrm{v}$ denotes the wavelet transform of the noise. The variances $\sigma_{*}^{2}, \sigma_{y}^{2}, \sigma_{v}^{z}, \mathrm{x}, \mathrm{v}$ and $y$ are given by,

$$
\sigma_{y}^{2}=\sigma_{v}^{2}+\sigma_{x}^{2}
$$


Step2: The noise standard derivation can be accurately estimated from the first decomposition level diagonal subbandD1 by the robust and accurate median estimator

$$
\sigma_{v}=\frac{\text { median }\left(\mid D_{1}\right) \mid}{0.6745}
$$

\section{EXPERIMENTAL RESULTS AND DISCUSSION}

On the behavioural study of the denoised images with respect to the following filters such as Wiener filter, Lucy-Richardson filter and Regularized filter the comparison of the PSNR shows that the Wiener filter is the suitable filter for denoising. Next, the denoised image is passed to the adaptive Wiener filter with optimal threshold from various shrinkage algorithms for nuclei segmentation. Comparison results based on threshold values and MSE for VisuShrink , BayesShrink and SureShrink Thresholding shows that adaptive wiener filter performs well with Sure shrink threshold when compared to the adopted other shrinkage methods. The following graph fig.3, fig.4, fig.5 and fig.6 shows the performance of the Wiener filter and the performance of adaptive wiener filter in combination with Sureshrink in terms of objective evaluation.

\begin{tabular}{|c|c|c|c|c|}
\hline \multirow{2}{*}{$\begin{array}{c}\text { Original } \\
\text { Images }\end{array}$} & \multirow{2}{*}{$\begin{array}{l}\text { Wiener } \\
\text { Filter }\end{array}$} & \multicolumn{3}{|c|}{ Adaptive Wiener Filter } \\
\hline & & $\begin{array}{l}\text { Visu } \\
\text { shrink }\end{array}$ & $\begin{array}{l}\text { Bayes } \\
\text { shrink }\end{array}$ & $\begin{array}{l}\text { Sure } \\
\text { shrink }\end{array}$ \\
\hline & 16. & & $\begin{array}{r}2 \\
-8\end{array}$ & \\
\hline $2^{5}$ & $x$ & & 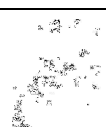 & \\
\hline & & & $t^{6}$ & \\
\hline 5. & 8 & to & & \\
\hline 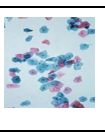 & $5^{2}$ & & 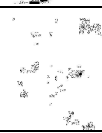 & s \\
\hline
\end{tabular}

Fig 2: Subjective comparison of adaptive wiener with visushrink threshold, adaptive wiener with Bayesshrink threshold and adaptive wiener with sureshrink threshold.

\subsection{PSNR (Peak Signal-to-Noise Ratio)}

For Pap smear images contaminated with Poisson noise, PSNR values can be calculated by comparing two images one is original image and other resultant image. The PSNR has been computed using the following formula

$$
P S N R=10 \log _{10\left[\frac{R^{2}}{M S E}\right]} \quad \cdots \cdots \cdots
$$

Where, $\mathrm{R}$ is the maximum variation in the input image data type. 


\subsection{MSE (Mean Squared Error)}

Mean Squared Error (MSE) is one way of measuring this similarity to compute an error signal by subtracting the test signal from the reference, and then to compute the average energy of the error signal.

\subsection{Threshold}

Threshold technique is one of the essential techniques in image segmentation. This technique can be expressed a

$$
T=T[x, y, p(x, y), f(x, y)]
$$

where, $T$ is the threshold value. $x, y$ are the coordinates of the threshold value point. $p(x, y), f(x, y)$ are points the gray level image pixels, Threshold image $\mathrm{g}(\mathrm{x}, \mathrm{y})$ can be define,

$$
g(x, y)= \begin{cases}1 & f(x, y)>T \\ 0 & f(x, y) \leq T\end{cases}
$$

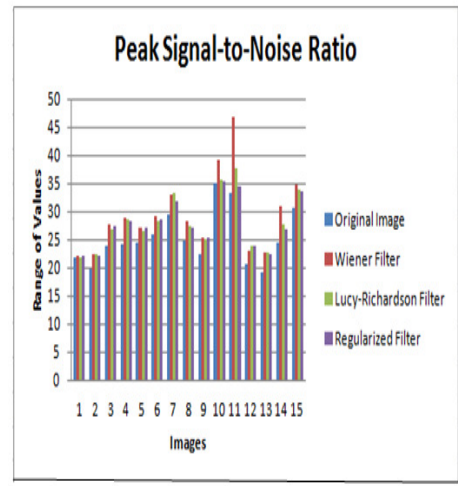

Fig.3: PSNR metric comparison results for Wiener filter, Lucy-Richardson filter and Regularized filter.

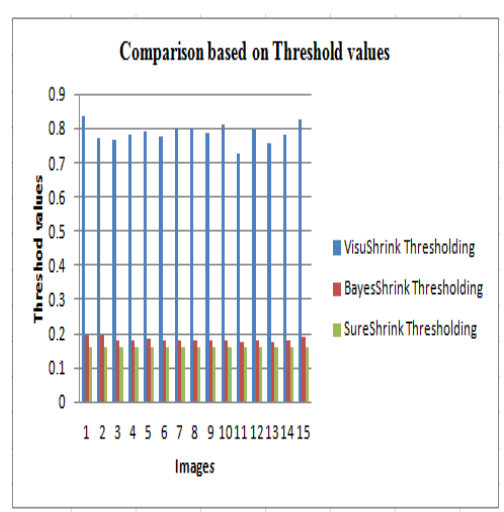

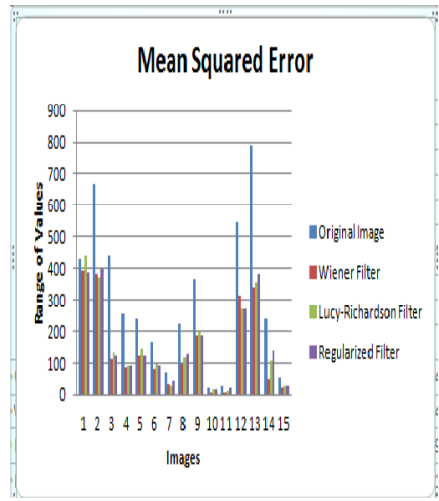

Fig.4: MSE metric comparison results for Wiener filter, Lucy-Richardson filter and Regularized filter.

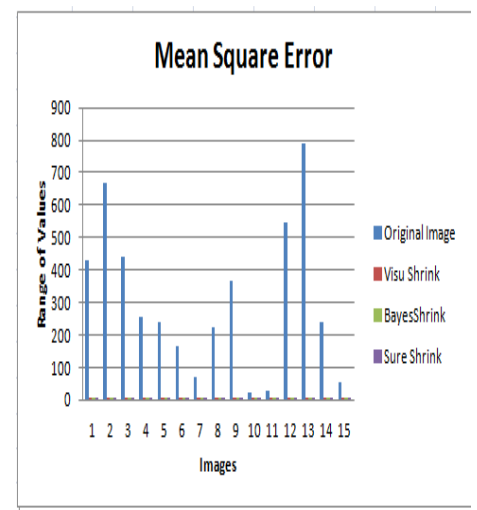

Fig.5, 6: PSNR and MSE metric comparison results for Adaptive Wiener filter with VisuShrink thresholding, Adaptive Wiener filter with BayesShrink thresholding and Adaptive Wiener filter with SureShrink thresholding. 


\section{CONCLUSIONS}

In this paper, denoising is performed on Pap smear images using Wiener filter, Lucy Richardson filter and Regularized filters and the Peak Signal-to-Noise Ratio (PSNR), Mean Squared Error (MSE) is calculated. After denoising by these filters, PSNR values and MSE values are compared and it is found that Wiener is more efficient than other filters for removing the Poisson noise in the Pap smear images and also it enhances the visual quality of the Pap smear images. Then, the nucleus is segmented from the denoised image with optimal threshold value obtained from the shrinkage algorithms like VisuShrink thresholding, BayesShrink thresholding and SureShrink thresholding. Based on the subjective results of threshold values and MSE it is confirmed that the performance of adaptive wiener filter in combination with Sure Shrink threshold gives the better result than the other adopted shrinkage methods. In future, based on the size of the segmented nucleus it is possible to differentiate abnormal cells from the normal cells in the Pap smear images.

\section{REFERENCES}

[1] Asmaa Abass Ajwad, 2012,'Noise Reduction of Ultrasound Image Using Wiener filtering and Haar Wavelet Transform Techniques", Diyala Journal of Medicine, Vol. 2, Issue 1, pp.91-100.

[2] E.Jebamalar Leavline, S.Sutha and D.Asir Antony Gnana Singh, 2011, "Wavelet Domain Shrinkage Methods for Noise Removal in Images: A Compendium”, International Journal of Computer Applications (0975 - 8887) Volume 33- No.10, pp.28-32.

[3] Iman Elyasi, 2012," Noise Removal and Enhancement Mammographic Images by Using Bayesshrink", ISSN 2090-4304, Journal of Basic and Applied Scientific Research, TextRoad Publication, J. Basic. Appl. Sci. Res., pp.2962-2965.

[4] Marina E.Plissiti, Christophoros Nikou, 2010, "Accurate Localization of Cell Nuclei in Pap smear im-ages using Gradient vector flow deformable models ”,BIOSIGNALS 2010, Internal Conference on Bio-inspired Systems and Signal Processing,pp.284-289.

[5] N.Lassouaoui, L.Hamami, and N.Nouali, 2005, "Morphological Description of Cervical Cell Images for the Pathological Recognition", Proceedings of world academy of science, Engineering and Technology, Volume-5, ISSN 1307-6884, pg-49-52

[6] Richardson, William Hadley, 1972, "Bayesian-Based Iterative Method of Image Restoration", JOSA 62, 10.1364/JOSA.62.000055, pp.55-59.

[7] S.Sudha, G.R.Suresh and R.Sukanesh, 2009, "Speckle Noise Reduction in Ultrasound Images by Wave-let Thresholding based on Weighted Variance", International Journal of Computer Theory, Vol. 1, No.1,pp.7-11.

[8] Shintaro Eday and Tetsuya Shimamura, 2007," Image Denoising for Poisson Noise by Pixel Values Based Division and Wavelet Shrinkage”, International Symposium on Nonlinear Theory and its Applications, NOLTA'07, pp.441-446.

[9] S. Grace chang, student member, ieee, bin yu, senior member, IEEE, and martin vetterli, fellow, IEEE, 2000,"Adaptive wavelet thresholding for image denoising and compression, ieee transac-tions on image processing", 00\$10.00 @ 2000 IEEE, vol. 9, no. 9, pp. 1057-7149.

[10] Sreedevi M T, Sandya S 2012, "Pap smear Image based Detection of Cervical Cancer", International Journal of Computer Applications (0975 - 8887) Volume 45- No.20, pp.35-40. 